\title{
Just so Higgs boson
}

\author{
F. Bazzocchi \\ IFIC - Instituto de Física Corpuscular University of Valencia, Apartado de Correos 22085 E-46071 Valencia, Spain \\ M. Fabbrichesi and P. Ullio \\ INFN, Sezione di Trieste and Scuola Internazionale Superiore di Studi Avanzati via Beirut 4, I-34014 Trieste, Italy
}

(Received 10 January 2007; published 23 March 2007)

\begin{abstract}
We discuss a minimal extension to the standard model in which there are two Higgs bosons and, in addition to the usual fermion content, two fermion doublets and one fermion singlet. The little hierarchy problem is solved by the vanishing of the one-loop corrections to the quadratic terms of the scalar potential. The electroweak ground state is therefore stable for values of the cut off up to $10 \mathrm{TeV}$. The Higgs boson mass can take values significantly larger than the current LEP bound and still be consistent with electroweak precision measurements.
\end{abstract}

DOI: 10.1103/PhysRevD.75.056004

PACS numbers: 11.30.Qc, 12.60.Fr, 14.80.Cp, 95.35.+d

\section{MOTIVATIONS}

There is some tension between the value of the electroweak (EW) vacuum and the scale at which we expect new physics to become manifest according to EW precision measurements [1]. If we take the latter scale (around $10 \mathrm{TeV}$ ) as the cut off of our effective theory, some degree of fine tuning is necessary in the scalar potential in order to guarantee the stability of the vacuum against radiative corrections. This little hierachy problem - and before it the more general (and more serious) problem of the large hierarchy between the EW vacuum and the GUT and Planck scale - has been used as a clue to the development of models in which the scalar sector of the standard model is enlarged to provide better stability, as, for instance, in supersymmetry, technicolor and little-Higgs models.

Here we discuss a different approach in which no new symmetry is introduced to cancel loop corrections and instead the parameters of the Lagrangian are such as to make the one-loop corrections vanish and thus ensure the stability of the effective potential for the scalar particles up to the energy scale at which two-loop effects begin to be sizable, namely, $10 \mathrm{TeV}$. Clearly, by its very nature, such a procedure can only be applied to the little hierarchy problem and not to the more general GUT or Planck-scale hierarchy problem. It is a limited solution to a little (hierarchy) problem, a problem that - contrary to those arising in much larger hierarchies - may well be contingent to the choice of the Lagrangian parameters.

Given the simplicity of the idea behind this approach, it is not surprising that it was suggested early on (by Veltman [2]) in the following terms: the quadratically divergent one-loop correction to the Higgs boson mass $m_{h}$,

$$
\frac{3 \Lambda^{2}}{16 \pi^{2} v_{W}^{2}}\left[2 m_{W}^{2}+m_{Z}^{2}+m_{h}^{2}-4 m_{t}^{2}\right]
$$

can be made to vanish, or at least made small enough, if $m_{h}$ happens to be around $316 \mathrm{GeV}$ at the tree level.
Contributions not included in (1) are proportional to the light quark masses and therefore negligible.

Such a cancellation does not originate from any dynamics and it is the accidental result of the values of the physical parameters of the theory. The absence of this quadratically divergent term in the two-point function of the scalar bosons makes possible to increase the cut off for the theory to a higher value with respect to that of the standard model (SM) where the renormalization of the Higgs boson mass and the given value of the expectation value $v_{W}$ impose a cut off of around $1 \mathrm{TeV}$ to avoid unnaturally precise cancellations among terms.

We now know that a value of $m_{h}=316 \mathrm{GeV}$ is a little over $3 \sigma$ with respect to current precision measurements of EW data [1]. This however does not mean that a scenario in which the Higgs boson mass is chosen just-so to make the cancellation $a ̀$ la Veltman is ruled out. It only means that we must either enlarge the SM with new particles propagating below $1 \mathrm{TeV}$ and then redo the EW data fit [3] or introduce new physics at a higher scale, the effect of which is to correct the precision observables and make room for the shifted value of the Higgs boson mass (as described in the framework of the effective EW Lagrangian in [4]).

Bearing this in mind, we introduce the minimal extension to the SM in which

(i) quadratically divergent contributions cancel at oneloop à la Veltman;

(ii) it is consistent with the EW precision data.

The model, as we shall see, is quite simple and provides an explicit example of an extension of the SM in which the mass of the Higgs boson can assume significantly larger values with respect to the current lower bound without having the EW precision measurements violated. In so doing, it introduces a characteristic spectrum of states beyond the SM that can be investigated at the LHC.

The model is natural in the sense that the EW vacuum is stable against a cut off of the order of $10 \mathrm{TeV}$ for a large choice of parameters. It is just-so because the physical 
parameters are chosen by hand in order to satisfy the constraints. These parameters are however numbers of order unity and not extravagantly small or large; moreover, they can be chosen among many possible values so that no unique determination is required, as it would be in the original Veltman's condition where the only free parameter is the Higgs boson mass, or-what amounts to the same thing - the quartic coupling $\lambda$.

Because among the additional states required there is a stable neutral (exotic) fermion, we also discuss to what extent this state can be considered a candidate for dark matter.

\section{THE MODEL: HOW THE HIGGS GOT ITS MASS}

We consider a model in which there are two scalar EW doublets, $h_{1}$ and $h_{2}$, the lightest scalar component of which is going to be identified with the SM Higgs boson and two Weyl fermion doublets, $\psi_{1}$ and $\psi_{2}$. In addition, we also introduce a Weyl fermion $\psi_{3}$ that is a $S U(2)_{W} \times U(1)_{Y}$ singlet.

Let us briefly discuss to what extent this choice of new states is the minimal extension which cancels the quadratic divergence. In the model with only two Higgs bosons it is possible to reduce - or indeed cancel - the contribution of the top quark to the quadratic divergence but not that of the gauge bosons and the cut off cannot be raised up to $10 \mathrm{TeV}$. We comment on this class of models in Sec. VII below. The mass of a single fermion doublet (with two singlets) is necessarily proportional to the scalar field vacuum expectation value (VEV) and cannot be varied independently of the Veltman condition (if we want to choose naturally the Yukawa of the new fermions). Two doublet fermions are also necessary in order to be anomaly free. The singlet fermion is necessary to lift the fermion degeneracy and couple the fermions to the scalar fields.

The states of the model are similar to those of a SUSY minimal extension of the scalar sector of the SM into a Wess-Zumino chiral model in which the singlet boson has been integrated out. However, a model with softly broken supersymmetry cannot be the model we are discussing because the supersymmetry, if present at any scale, would make the quadratic divergence zero.

The Lagrangian for the scalar bosons is given by

$$
\mathcal{L}_{h}=\sum_{i=1,2} D_{\mu} h^{i} D^{\mu} h^{i}+V\left[h_{1}, h_{2}\right]
$$

with the potential

$$
\begin{aligned}
V\left[h_{1}, h_{2}\right]= & \lambda_{1}\left(h_{1}^{\dagger} h_{1}\right)^{2}+\lambda_{2}\left(h_{2}^{\dagger} h_{2}\right)^{2}+\lambda_{3}\left(h_{1}^{\dagger} h_{1}\right)\left(h_{2}^{\dagger} h_{2}\right) \\
& +\lambda_{4}\left(\tilde{h}_{2}^{\dagger} h_{1}\right)\left(\tilde{h}_{1}^{\dagger} h_{2}\right)+\lambda_{5}\left[\left(\tilde{h}_{2}^{\dagger} h_{1}\right)^{2}+\text { H.c. }\right] \\
& +\mu_{1}^{2}\left(h_{1}^{\dagger} h_{1}\right)+\mu_{2}^{2}\left(h_{2}^{\dagger} h_{2}\right),
\end{aligned}
$$

where $\tilde{h}_{2}=i \sigma_{2} h_{2}^{*}$. The potential in Eq. (3) is the most general for the two Higgs doublets once we impose a parity symmetry $T_{1}$ according to which the two doublets $h_{1}$ and $h_{2}$ are, respectively, even and odd. In this way, the quadratic and quartic mixing terms are forbidden, which makes the discussion simpler.

The ground state that triggers the electroweak symmetry breaking. It is

$$
\left\langle h_{1}\right\rangle=\left(\begin{array}{c}
0 \\
v_{1} / \sqrt{2}
\end{array}\right) \text { and }\left\langle h_{2}\right\rangle=\left(\begin{array}{c}
v_{2} / \sqrt{2} \\
0
\end{array}\right) .
$$

The requirement of matching the EW vacuum $v_{w}$ to this vacuum state constrains one parameter of the model.

The mass eigenstates of the scalar particles can thus be derived. The masses are

$$
\begin{aligned}
m_{h, H}^{2}= & \lambda_{1} v_{1}^{2}+\lambda_{2} v_{2}^{2} \\
& \pm \sqrt{\left(\lambda_{1} v_{1}^{2}-\lambda_{2} v_{2}^{2}\right)^{2}+\left(\lambda_{3}+\lambda_{4}+\lambda_{5}\right)^{2} v_{1}^{2} v_{2}^{2}} \\
m_{A}^{2}= & -\lambda_{5} v_{w}^{2},
\end{aligned}
$$

for the three neutral scalar bosons (two of which, $h$ and $H$, are scalars and one, $A$, a pseudoscalar),

$$
m_{H^{+}}^{2}=-\left(\lambda_{4}+\lambda_{5}\right) v_{w}^{2},
$$

for the charged boson $\mathrm{H}^{+}$after using the constraint $v_{w}^{2}=$ $\left(v_{1}^{2}+v_{2}^{2}\right)$ in Eqs. (5) and (6).

By introducing the mixing angle $\alpha$ and $\beta$ to rotate the scalar boson gauge states into the mass eigenstates, we write:

$$
\begin{aligned}
& h_{1}=\frac{1}{\sqrt{2}}\left(\begin{array}{c}
\sin \beta H^{+} \\
v_{1}+\cos \alpha h-\sin \alpha H+\sin \beta A
\end{array}\right) \text { and } \\
& h_{2}=\frac{1}{\sqrt{2}}\left(\begin{array}{c}
v_{2}+\sin \alpha h+\cos \alpha H+\cos \beta A \\
\cos \beta H^{-}
\end{array}\right) .
\end{aligned}
$$

As usual, in 2 Higgs doublet model $\tan \beta=v_{2} / v_{1}$ and

$$
\tan 2 \alpha=\frac{\lambda_{3} v_{1} v_{2}}{\left(\lambda_{2} v_{2}^{2}-\lambda_{1} v_{1}^{2}\right)}
$$

The exotic fermion content of the model is given by two $S U(2)_{W}$ doublets:

$$
\Psi_{1}=\left(\begin{array}{c}
\psi_{1}^{+} \\
\psi_{1}^{0}
\end{array}\right) \quad \Psi_{2}=\left(\begin{array}{c}
\psi_{2}^{0} \\
\psi_{2}^{-}
\end{array}\right),
$$

and one $S U(2)_{W}$ singlet $\psi_{3}$; we can also define the Majorana 4-components fermions current eigenstates as

$$
\tilde{\psi}_{i}^{0}=\left(\begin{array}{c}
\psi_{i}^{0} \\
\bar{\psi}_{i}^{0}
\end{array}\right) \quad \tilde{\chi}_{i}^{+}=\left(\begin{array}{l}
\psi_{1}^{+} \\
\bar{\psi}_{2}^{-}
\end{array}\right) .
$$

The SM fermions are even under the $T_{1}$ parity symmetry and therefore can have Yukawa interactions only with the scalar doublet $h_{1}$. The exotic doublet fermions $\Psi_{i}$ are odd under this parity symmetry while the singlet $\psi_{3}$ is even. We also introduce an additional parity $T_{2}$ under which the 
Higgs bosons are even while all the exotic fermions are odd (SM particles are always even under both parities).

In this way the exotic fermions do not mix with the SM fermions and may have Yukawa terms only with the scalar doublet $h_{2}$. No new processes are generated except in the decay of the top where however the current precision in not sufficient in providing additional constraints. Flavor physics can only be affected by these new fermions in diagrams with two or more loops and therefore we do not expect new constraints to the parameters of the model.

The Lagrangian for the exotic fermions is simply given by the kinetic and the Yukawa terms, that is

$$
\mathcal{L} \psi=\mathcal{L}_{\text {kin }}+\mathcal{L}_{m}^{\psi}
$$

where $\mathcal{L}_{\text {kin }}$ is given by

$$
\mathcal{L}_{\text {kin }}=\bar{\Psi}_{1} \hat{D} \Psi_{1}+\bar{\Psi}_{2} \hat{D} \Psi_{2}+\bar{\psi}_{3} \hat{\partial} \psi_{3}
$$

and

$$
\begin{aligned}
-\mathcal{L}_{m}= & \tilde{\mu} \epsilon_{i j} \Psi_{1_{i}} \Psi_{2_{j}}+\hat{\mu} \psi_{3} \psi_{3}+\left(\frac{k_{1}}{\sqrt{2}} \psi_{3}{\tilde{h_{2}}}^{\dagger} \Psi_{1}+\text { H.c. }\right) \\
& +\left(\frac{k_{4}}{\sqrt{2}} \psi_{3} \Psi_{2_{i}} \tilde{h_{2 j}}+\text { H.c. }\right) .
\end{aligned}
$$

From $\mathcal{L}_{m}$ we see that the charged Dirac fermion $\tilde{\chi}^{+}$has mass $m_{\chi^{+}}=\tilde{\mu}$ while once we insert Eq. (4) into Eq. (9) the Majorana mass matrix for the neutral states is given by

$$
M^{0}=\left(\begin{array}{ccc}
0 & -\tilde{\mu} & k_{1} v_{2} / \sqrt{2} \\
-\tilde{\mu} & 0 & k_{4} v_{2} / \sqrt{2} \\
k_{1} v_{2} / \sqrt{2} & k_{4} v_{2} / \sqrt{2} & \hat{\mu} .
\end{array}\right)
$$

This matrix is diagonalized by a neutralino mixing matrix $V$ which satisfies $V^{T} M^{0} V^{*}=M_{\text {diag }}^{0}$. From the 2components mass eigenvectors of Eq. (10) $\chi_{i=1,3}^{0}$, we define the 4-components neutral fermions that are our neutralinos:

$$
\tilde{\chi}_{1}^{0}=\left(\begin{array}{c}
\chi_{1}^{0} \\
\bar{\chi}_{1}^{0}
\end{array}\right), \quad \tilde{\chi}_{2}^{0}=\gamma_{5}\left(\begin{array}{c}
\chi_{2}^{0} \\
\bar{\chi}_{2}^{0}
\end{array}\right), \quad \tilde{\chi}_{3}^{0}=\left(\begin{array}{c}
\chi_{3}^{0} \\
\bar{\chi}_{3}^{0}
\end{array}\right),
$$

where in Eq. (11) the definition of $\tilde{\chi}_{2}^{0}$ takes into account that the corresponding eigenvalue of the Majorana mass matrix is negative.

From $\mathcal{L}_{\text {kin }}$ of Eq. (9) using the mass eigenstates defined in Eq. (11) we obtain the interaction terms of the new fermions with the gauge bosons

$$
\begin{aligned}
\mathcal{L}= & \frac{g}{\sqrt{2}}\left[\sum_{i=1,3} \overline{\tilde{\chi}}^{+} \gamma_{\mu}\left(V_{1 i} \epsilon_{i} P_{L}-V_{2 i}^{*} P_{R}\right) \tilde{\chi}_{i}^{0}\right] W^{+\mu} \\
& + \text { H.c. }+\frac{g}{2} \overline{\tilde{\chi}}^{+} \gamma_{\mu}\left(P_{L}+P_{R}\right) \tilde{\chi}^{+} W_{3}^{\mu} \\
& -\frac{g}{2} \sum_{i, j=1,3}\left[\overline { \tilde { \chi } } _ { i } ^ { 0 } \gamma _ { \mu } \left(\epsilon_{i} \epsilon_{j}\left(V_{i 1}^{\dagger} V_{1 j}-V_{i 2}^{\dagger} V_{2 j}\right) P_{L}\right.\right. \\
& \left.\left.-P_{R}\left(V_{i 1}^{T} V_{1 j}^{*}-V_{i 2}^{T} V_{2 j}^{*}\right)\right) \tilde{\chi}_{j}^{0}\right] W_{3}^{\mu} \\
& +\frac{g^{\prime}}{2} \overline{\tilde{\chi}}^{+} \gamma_{\mu}\left(P_{L}+P_{R}\right) \tilde{\chi}^{+} B^{\mu} \\
& +\frac{g^{\prime}}{2} \sum_{i, j=1,3}\left[\overline { \tilde { \chi } } _ { i } ^ { 0 } \gamma _ { \mu } \left(\epsilon_{i} \epsilon_{j}\left(V_{i 1}^{\dagger} V_{1 j}-V_{i 2}^{\dagger} V_{2 j}\right) P_{L}\right.\right. \\
& \left.\left.-P_{R}\left(V_{i 1}^{T} V_{1 j}^{*}-V_{i 2}^{T} V_{2 j}^{*}\right)\right) \tilde{\chi}_{j}^{0}\right] B^{\mu},
\end{aligned}
$$

where the factor $\epsilon_{i}=(-1)^{i-1}$ keeps into account the signs of the eigenvalues of the Majorana mass matrix of Eq. (10). This Lagrangian is necessary in order to compute the oneloop radiative corrections to the scalar potential.

\section{VELTMAN CONDITION REDUX}

As stated in the introduction, we want to stabilize the potential given by Eq. (3) at one-loop level, that is we want the one-loop quadratically divergent contributions to $\mu_{i}^{2}$ to be zero. As in the SM the quadratically divergent contributions arise by loops of gauge bosons, scalars and fermions. We therefore find two Veltman conditions by imposing

$$
\delta \mu_{1}^{2}=0 \quad \text { and } \quad \delta \mu_{2}^{2}=0,
$$

that is

$$
\begin{array}{r}
\frac{9}{4} g^{2}+\frac{3}{4} g^{\prime 2}+2\left(3 \lambda_{1}+\lambda_{3}+\lambda_{4}\right)-12 \lambda_{t}^{2}=0 \\
\frac{9}{4} g^{2}+\frac{3}{4} g^{\prime 2}+2\left(3 \lambda_{2}+\lambda_{3}+\lambda_{4}\right)-\left(k_{1}^{2}+k_{4}^{2}\right)=0 .
\end{array}
$$

In Eq. (14) $g, g^{\prime}$ are the electroweak gauge couplings, $\lambda_{i}$ the parameter of the scalar potential of Eq. (3), $\lambda_{t}$ the top Yukawa defined as $\lambda_{t}=v_{w} / v_{1}$ since the SM fermions couple only to the scalar doublet $h_{1}$ and $k_{1,4}$ are the Yukawa coupling of Eq. (9). The contributions of the lighter SM fermions to Eq. (14) have been neglected.

Notice that if we did not have the parity symmetry $T_{1}$ and the fermions had interacted with both $h_{1}$ and $h_{2}$ we would have generated a divergent mixed contribution that could have been canceled only by a bare term.

In writing Eq. (14) we have taken a common cut off $\Lambda$ for the divergent loops of different states. The possibility that there exist different cutoffs for the different contributions does not change our result because a change of order one in the $\Lambda \mathrm{s}$ only means a similar change of order one in the parameters of the model $\lambda_{i}$ and $k_{i}$.

Once these two conditions are satisfied the scalar potential is stable at one-loop order and so is its vacuum state. 
We interpret these conditions as two constraints on the 10 free parameters of the model.

\section{THE EW PARAMETERS $S, T$ AND $U$}

For our purposes, the consistence of the model against EW precision measurements can be checked by means of oblique corrections. These corrections can be classified [5] by means of three parameters:

$$
\begin{aligned}
\alpha S & =-4 e^{2}\left[\Pi_{33}^{\prime}(0)-\Pi_{3 B}^{\prime}(0)\right] \\
\alpha T & =\frac{e^{2}}{s_{W}^{2} c_{W}^{2} m_{Z}^{2}}\left[\Pi_{11}(0)-\Pi_{33}(0)\right] \\
\alpha U & =4 e^{2}\left[\Pi_{11}^{\prime}(0)-\Pi_{33}^{\prime}(0)\right],
\end{aligned}
$$

where the functions $\Pi_{n m}\left(q^{2}=0\right)$ represent the vacuum polarizations of the gauge vectors in the various directions of isospin space. Other corrections functions-like the functions $Y$ and $W$ of Ref. [6] - are not relevant here because mainly sensitive to physics in which there are new vector bosons.

EW precision measurements severely constrain the possible values of the three parameters $\mathrm{S}, \mathrm{T}$ and $\mathrm{U}$. In the SM, the data allow [1], for a Higgs boson mass of $m_{h}=$ $117 \mathrm{GeV}$,

$$
\begin{gathered}
S=-0.13 \pm 0.10 \quad T=-0.17 \pm 0.12 \\
U=0.22 \pm 0.13 .
\end{gathered}
$$

These constraints must be rescaled for the different values of the Higgs boson mass.

If we want the model to be consistent with the EW precision measurements within, for instance, one sigma we have three further constraints on the parameters of the model -5 of which still remain free at this point.

A mass of the Higgs boson larger than the reference value will make the parameter $T$ smaller, the size of the correction going like $\ln m_{h}^{2} / m_{Z}^{2}$. This can be compensated by the fermion contribution which can give a $\Delta T>0$ of size $\Delta m^{2} \ln m^{2}$ where $\Delta m^{2}$ is the isospin splitting of the fermion masses. The parameter $S$ is changed by the larger Higgs mass with $\Delta S>0$, a change that is in general difficult to compensate. In our model a negative contribution to $S$ comes about because of the fermion with both Dirac and Majorana masses which give a negative contribution proportional to $\ln \left(m_{\chi^{+}} / m_{\chi_{i}^{0}}\right)$, where $m_{\chi^{+}}-m_{\chi_{i}^{0}}$ is the isospin mass splitting between the chargino and the neutralino $i$.

Let us consider scalar and fermion contributions to $T$ and $S$ separately in a simplified model which helps in visualizing better how the contributions compensate one other in order to accommodate the EW experimental values.

For what concerns the fermions, suppose to be in the simple case in which $m_{\chi^{+}} \sim m_{\chi_{i}^{0}}$. The fermion contribu- tion to the $T$ parameter can be written as

$$
T^{f}=T_{L L}^{f}+T_{L R}^{f},
$$

where $T_{L L}^{f}$ takes into account the one-loop contributions that arise from the vacuum polarizations of the gauge bosons of the kind $\Pi_{11,33}^{L L}$ and $\Pi_{11,33}^{R R}, T_{L R}^{f}$ the ones that arise from $\Pi_{11,33}^{L R}$ and $\Pi_{11,33}^{R L}$. The latter are not present in the SM case. Keeping only the leading contributions, we have

$$
\begin{aligned}
T_{L L}^{f}= & \frac{2}{c_{W}^{2} s_{W}^{2} m_{Z}^{2} \pi}\left\{\sum_{i} U_{i}^{\mathrm{eff}}\left[-m_{\chi^{+}}^{2}+\frac{\left(m_{\chi^{+}}-m_{\chi_{i}}\right)^{2}}{2}\right] \log \frac{m_{\chi^{+}}^{2}}{m_{Z}^{2}}\right. \\
& \left.-\sum_{i, j} \frac{U_{i j}^{\mathrm{eff}}}{2}\left[-m_{\chi_{i}^{0}}^{2}+\frac{\left(m_{\chi_{i}^{0}}-m_{\chi_{j}^{0}}\right)^{2}}{2}\right] \log \frac{m_{\chi_{i}^{0}}^{2}}{m_{Z}^{2}}\right\} \\
T_{L R}^{f}= & \frac{2}{c_{W}^{2} s_{W}^{2} m_{Z}^{2} \pi}\left\{\sum_{i} U_{i}^{\mathrm{eff}} m_{\chi^{+}} m_{\chi_{i}^{0}} \log \frac{m_{\chi^{+}}^{2}}{m_{Z}^{2}}\right. \\
& \left.-\sum_{i, j} \frac{U_{i j}^{\mathrm{eff}}}{2} m_{\chi_{j}^{0}} m_{\chi_{i}^{0}} \log \frac{m_{\chi_{i}^{0}}^{2}}{m_{Z}^{2}}\right\}
\end{aligned}
$$

where $U_{i, i j}^{\text {eff }}$ are effective couplings related to the neutralino mixing matrix $V$ and are in general different for the $L L$ contribution and for the $L R$ one. For example $U_{i}^{\text {eff }}$ in $T_{L L}^{f}$ is given by $\Sigma_{k}=V_{i k}^{\dagger} V_{k i}$. Notice that when the third neutralino decouples, the $T_{L L}^{f}$ contribution goes to zero when $m_{\chi^{+}}=m_{\chi_{1,2}^{0}}$ and the isospin symmetry is restored. The same happens also for $T_{L R}^{f}$. In a similar manner, the $S$ parameter receives a fermion contribution that can be split in

$$
S^{f}=S_{L L}^{f}+S_{L R}^{f},
$$

with

$S_{L L}^{f}=\frac{1}{3 \pi}\left[\left(U_{i j}^{\mathrm{eff}}-1\right)-2 \log \frac{m_{\chi^{+}}^{2}}{m_{Z}^{2}}+2 U_{i j}^{\mathrm{eff}} \log \frac{m_{\chi_{i}^{0}}^{2}}{m_{Z}^{2}}\right]$

$S_{L R}^{f}=\frac{2}{3 \pi}\left(y_{R_{\chi^{+}}}-y_{R_{\chi_{i j}^{0}}}^{\mathrm{eff}}\right)$,

where $y_{R_{\chi^{+}}}=1 / 2$ follows by the definition of $\chi^{+}$and where we have defined $y_{R_{\chi_{i j}^{0}}}^{\text {eff }}=2 \Sigma_{k=1,2} T_{3_{k}} y_{R_{k}} f\left(V_{k i}, V_{k j}\right)$ where $T_{3_{k}}$ and $y_{R_{k}}$ are the isospin and the hypercharge of the Majorana singlets defined in Eq. (8) and $f\left(V_{k i}, V_{k j}\right)$ is a combination of different entries of the neutralino mixing matrix $V$. Notice that we recover the contribution of two SM-like doublets when the hypercharges difference in $S_{L R}^{f}$ gives $1 / 2$ and $U_{i j}$ in $S_{L L}^{f}$ is equal to 1 [5].

The previous expressions can be further simplified if we consider the fermion mass matrix of Eq. (10) in the limit in which $\hat{\mu}$ is much larger than $\tilde{\mu}$ and $k_{1,4} v_{2}$. In this limit the neutral fermion mixing matrix is approximately given by 



FIG. 1 (color online). Fermion contributions to the parameters $T$ and $S$ as a function of their mass splitting. The plots are made for one representative value of the $\chi_{3}^{0}$ mass.

$$
V=\left(\begin{array}{ccc}
-\frac{1}{\sqrt{2}} & -\frac{1}{\sqrt{2}} & \sqrt{\frac{m_{\chi_{2}^{0}}-m_{\chi_{1}^{0}}}{2 m_{\chi_{3}^{0}}}} \\
\frac{3 m_{\chi_{1}^{0}}-m_{\chi_{2}^{0}}}{2 \sqrt{2} m_{\chi_{1}^{0}}} & -\frac{m_{\chi_{1}^{0}}+m_{\chi_{2}^{0}}}{2 \sqrt{2} m_{\chi_{1}^{0}}} & -\frac{m_{\chi_{3}^{0}}}{m_{\chi_{1}^{0}}} \sqrt{\frac{m_{\chi_{2}^{0}}-m_{\chi_{1}^{0}}}{2 m_{\chi_{3}^{0}}}} \\
\frac{m_{\chi_{1}^{0}}+m_{\chi_{3}^{0}}}{m_{\chi_{1}^{0}}} \sqrt{\frac{m_{\chi_{2}^{0}}-m_{\chi_{1}^{0}}}{2 m_{\chi_{3}^{0}}}} \frac{m_{\chi_{1}^{0}}-m_{\chi_{3}^{0}}}{m_{\chi_{1}^{0}}} \sqrt{\frac{m_{\chi_{2}^{0}}-m_{\chi_{1}^{0}}}{2 m_{\chi_{3}^{0}}}} & 1
\end{array}\right) .
$$

If it holds also that $\tilde{\mu}$ is larger than $k_{1,4} v_{2}$ and $m_{\chi^{+}} \simeq m_{\chi_{1,2}^{0}}$, $T^{f}$ and $S^{f}$ can be easily expressed in terms of the mass splitting, see Fig. 1. Notice that Eq. (21) is valid for $m_{\chi_{1,2}^{0}}<$ $m_{\chi_{3}^{0}}<m_{\chi_{1}^{0}}^{2} / \Delta m_{\chi_{12}^{0}}$. For this reason we have plotted $T^{f}$ and $S^{f}$ in Fig. 1 corresponding to only one value of $m_{\chi_{3}^{0}}$, since in the range allowed differences are minimal.

For what concerns the scalar sector, consider the case in which $m_{H^{+}} \simeq m_{A}$. In this limit, $T^{s}$ and $S^{s}$ assume a simple form and we have

$$
T^{s}=-\frac{3}{16 \pi c_{W}^{2}}\left(\cos ^{2} \alpha \log \frac{m_{h}^{2}}{m_{Z}}+\sin ^{2} \alpha \log \frac{m_{H}^{2}}{m_{Z}}\right),
$$

where $\alpha$ is the mixing angle in the neutral scalar sector and

$$
S^{s}=\frac{1}{12 \pi}\left(\log \frac{m_{h}^{2}}{m_{Z}^{2}}+\log \frac{m_{H}^{2}}{m_{Z}^{2}}-2 \log \frac{m_{H^{+}}^{2}}{m_{Z}^{2}}+\log \frac{m_{A}^{2}}{m_{Z}^{2}}\right) .
$$

We can compare the contribution of the scalar sector of our

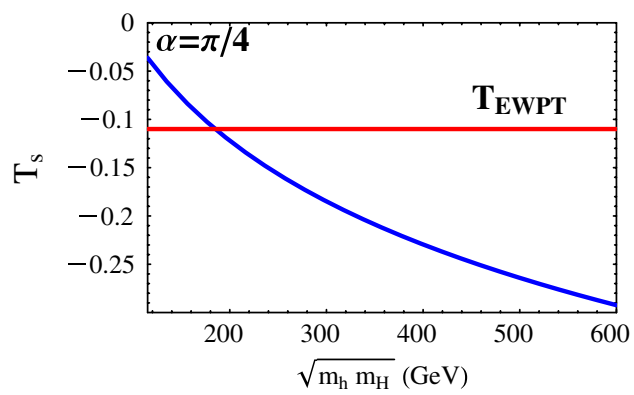

model with respect to the SM one. In the SM we have

$$
T_{h}^{S M}=-\frac{3}{8 \pi c_{W}^{2}} \log \frac{m_{h}}{m_{Z}} \quad \text { and } \quad S_{h}^{S M}=\frac{1}{12 \pi} \log \frac{m_{h}^{2}}{m_{Z}^{2}} .
$$

EW precision measurements indicate that at $2 \sigma m_{h} \leq$ $185 \mathrm{GeV}$ [1] and therefore the introduction of the fermions in our model is justified if $T^{s}$ and $S^{s}$ exceeds the contributions $T_{h}^{S M}$ and $S_{h}^{S M}$ corresponding to $m_{h} \simeq 185 \mathrm{GeV}$. This is shown in Fig. 2.

For fixed $m_{h}$ and $m_{H}$, and a given fermion spectrum that accommodates the $T$ parameter, the fermion contribution to $S$ is fixed and therefore the only freedom left is in the values of $m_{A}$ and $m_{H^{+}}$. In the case in which $m_{H^{+}} \simeq m_{A}$, their total contribution to $S$ has the same sign of the fermion one, therefore we expect that $m_{A}$ cannot in general be to heavy. This is verified in the numerical analysis.

\section{A DARK MATTER CANDIDATE?}

The lightest neutral exotic fermion state in the model is similar to the neutralinos in a minimal supersymmetric extension of the SM (NMSSM) in which the composition is dominated by Higgsinos. It is stable because the Lagrangian does not contain couplings between the SM and the exotic fermions - or, alternatively, you can think of the Lagrangian as written with a underlying conserved parity.

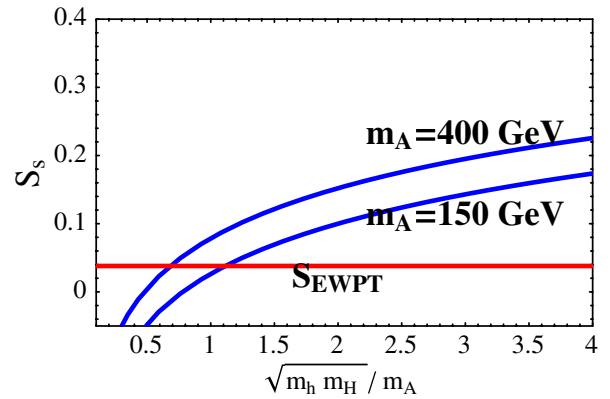

FIG. 2 (color online). Scalar contributions to the parameters $T$ and $S$ as a function of their masses. The red horizontal lines show the central value of the current EW bounds. Notation and values of the parameters are explained in the text. 
We compute by means of the program DARKSUSY [7] its relic abundance $\Omega_{D M} h^{2}$. To do this we need the Lagrangian written on the exotic fermion mass eigenstates:

$$
\begin{aligned}
-2 \mathcal{L}_{m}= & \sum_{i=1,2,3} m_{i} \overline{\tilde{\chi}}_{i}^{0} \chi_{i}^{0}+\sum_{i, j=1,2,3} \overline{\tilde{\chi}}_{i}^{0}\left(V_{i 3}^{T} V_{1 j} \epsilon_{j} P_{L}+V_{i 3}^{\dagger} V_{1 j}^{*} \epsilon_{i} P_{R}\right) \tilde{\chi}_{j}^{0}\left[\sum_{n=1,2}\left(k_{1} U_{2 n}^{R}+k_{2} U_{1 n}^{R}\right) H_{n}\right]+\sum_{i, j=1,2,3} \overline{\tilde{\chi}}_{i}^{0}\left(V_{i 3}^{T} V_{2 j} \epsilon_{j} P_{L}\right. \\
& \left.+V_{i 3}^{\dagger} V_{2 j}^{*} \epsilon_{i} P_{R}\right) \tilde{\chi}_{j}^{0}\left[\sum_{n=1,2}\left(k_{4} U_{2 n}^{R}+k_{3} U_{1 n}^{R}\right) H_{n}\right]+\sum_{i, j=1,2,3} \overline{\tilde{\chi}}_{i}^{0}\left[\left(-i V_{i 3}^{T} V_{1 j} \epsilon_{j} P_{L}+i V_{i 3}^{\dagger} V_{1 j}^{*} \epsilon_{i} P_{R}\right)\left(k_{1} \cos \beta+k_{2} \sin \beta\right)\right. \\
& \left.+\left(i V_{i 3}^{T} V_{2 j} \epsilon_{j} P_{L}-i V_{i 3}^{\dagger} V_{2 j}^{*} \epsilon_{i} P_{R}\right)\left(k_{4} \cos \beta+k_{3} \sin \beta\right)\right] \tilde{\chi}_{j}^{0} A+\overline{\tilde{\chi}}_{i}^{0} \tilde{\chi}^{+}\left(V_{i 3}^{T} P_{L}+V_{i 3}^{\dagger} \epsilon_{i} P_{R}\right)\left(\left(k_{2}-k_{3}\right) U_{12}^{C}\right. \\
& \left.+\left(k_{4}-k_{1}\right) \cos \beta\right) H^{-}+\text {H.c. }
\end{aligned}
$$

where $H_{n=1,2}=h, H$ are the two neutral scalars, $A$ the pseudoscalar, $H^{-}$the charged scalar, $\beta$ the mixing angle defined in Eq. (7) and $U^{R}$ is the mixing matrix related to the real neutral components of the two doublets $h_{1}$ and $h_{2}$ given by

$$
U^{R}=\left(\begin{array}{cc}
\cos \alpha & -\sin \alpha \\
\sin \alpha & \cos \alpha
\end{array}\right),
$$

where $\alpha$ is the mixing angle defined in Eq. (8).

The analysis shows that the relic abundance is always at least 1 order of magnitude too small than the presently favorite abundance of dark matter in the Universe. This seems to be due to the lack of cancellations among different diagrams introduced by the arbitrariness in the Yukawa couplings that makes pair annihilation rates too large. Therefore, the lightest neutral exotic fermion can at most be a marginal component of dark matter.

\section{THE MODEL SOLVED}

The model has 11 parameters, 10 of which are in principle free once the ground state has been identified with $v_{W}$. If we enforce the Veltman conditions - and thus make the one-loop quadratically divergent corrections vanishwe are left with eight parameters. These can be exchanged

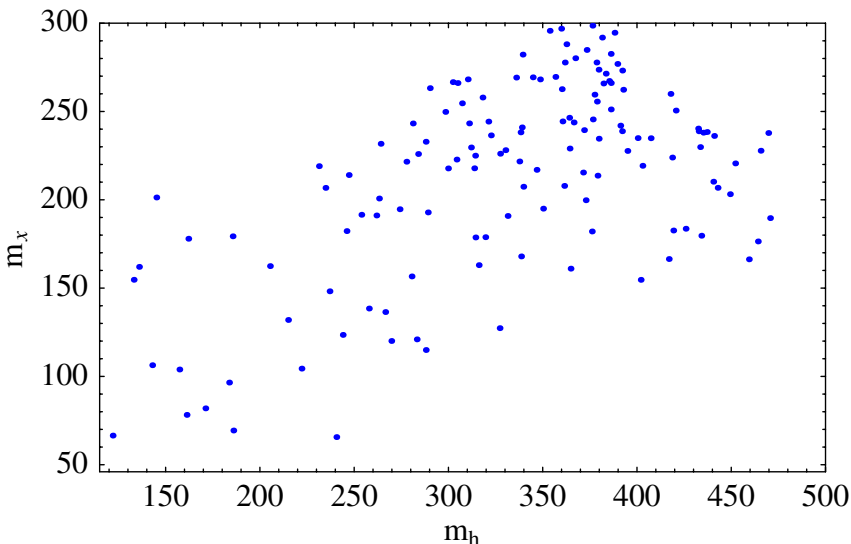

FIG. 3 (color online). Distribution of values for the masses $m_{\chi}$ vs $m_{h}$ for values of the parameters within $1 \sigma$ of EW precision measurements. for the masses of the 4 scalar and 4 fermion states. These can be varied and for each choice of them the $S, T$ and $U$ parameters computed and compared against the EW constraints.

We vary the dimensionless parameters within 1 order of magnitude. In particular, we keep the $\lambda_{i}$ and the $\kappa_{i}$ between 1 and $4 \pi$ (after which the perturbative analysis may break down). Mass parameters $\mu$ and $\tilde{\mu}$ are varied between 100 and $300 \mathrm{GeV}$.

We find that for a large choice of the five remaining parameters the model is consistent with the EW precision measurements. For these choices, masses as large as $450 \mathrm{GeV}$ are possible for the lightest neutral scalar Higgs boson. As $m_{h}$ increases those of the neutral pseudoscalar tend to favor lighter values so that there are solutions in which the lightest Higgs boson is the pseudoscalar. The lightest neutral fermion mass tends to increase together with the mass of the Higgs boson.

Figure 3 shows some of the possible values we obtain for the Higgs boson and lightest neutral fermion masses for values of the parameters which satisfy within $1 \sigma$ the EW precision measurements. Figure 4 shows the distribution of the masses for the scalar and pseudoscalar states under the same conditions.

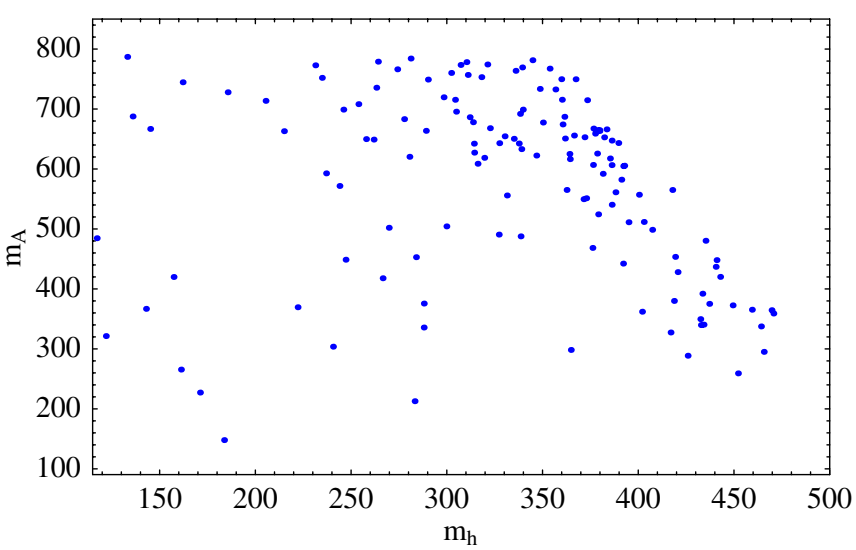

FIG. 4 (color online). Distribution of values for the masses $m_{A}$ vs $m_{h}$ for values of the parameters within $1 \sigma$ of EW precision measurements. 
TABLE I. Representative values (among those used in the plots) of the eight parameters of the model, and mass spectrum of the most relevant states: scalar and pseudoscalar bosons and lightest fermion, that satisfy the bounds from EW precision measurements.

\begin{tabular}{lc|rrrrrr|rrr}
\hline \hline$\mu(\mathrm{GeV})$ & $\tilde{\mu}(\mathrm{GeV})$ & $k_{1}$ & $\lambda_{1}$ & $\lambda_{2}$ & $\lambda_{3}$ & \multicolumn{1}{c|}{$\lambda_{4}$} & \multicolumn{1}{c|}{$\lambda_{5}$} & $m_{h}(\mathrm{GeV})$ & $m_{A}(\mathrm{GeV})$ & $m_{\chi}(\mathrm{GeV})$ \\
\hline 173 & 287 & 1.4 & 8.5 & 4.6 & 2.8 & -8.5 & -5.6 & 146 & 600 & 131 \\
138 & 128 & 1.6 & 6.3 & 6.4 & 2.3 & -10.6 & -2.9 & 210 & 417 & 96 \\
276 & 438 & 2.9 & 7.7 & 5.0 & 8.1 & -7.1 & -8.5 & 304 & 715 & 223 \\
266 & 381 & 3.8 & 5.3 & 12.5 & 1.7 & -7.0 & -3.5 & 450 & 460 & 212 \\
239 & 180 & 3.8 & 4.8 & 11.4 & 7.3 & -11.9 & -2.1 & 470 & 360 & 190 \\
\hline \hline
\end{tabular}

Our result may help in dispelling excessive surprise in not seeing a bantamweight Higgs boson with $m_{h}$ just above the current LEP bound of $117 \mathrm{GeV}$ and should encourage searches at the LHC for a Higgs boson substantially heavier than the current LEP bound-what we can call a welterweight $m_{h}$ around $300 \mathrm{GeV}$ or even a cruiserweight at $500 \mathrm{GeV}$. A similar scenario has been pointed out recently in $[8,9]$ in the framework of the little-Higgs models [10] and in [11,12] in a two-Higgs extension of the SM. Table I summarizes representative values of the eight parameters of the model and the mass spectrum of the most relevant states.

\section{MODELS WITH TWO HIGGS BOSONS AND NO EXTRA FERMIONS}

Different possibilities of realizing a minimal extension of the scalar sector of the SM with a natural cut off $\Lambda$ around few $\mathrm{TeV}$ and compatible with EW precision measurements have been discussed in the last year. The authors of [11-13] have analyzed different realizations of the 2 Higgs doublets model (2HDM) and have parametrized the fine tuning parameter in terms of the dependence of the mass of the light Higgs boson on the cut off $\Lambda$. In the Barbieri-Hall (BH) model [11] both doublets acquire a $\mathrm{VEV}$, but the small mixing angle between them makes the light scalar coupling to the top quark quite small and $\Lambda$ becomes proportional to the mass of the heavy neutral scalar. The mass of the heavy neutral scalar is then bounded by the requirement of satisfying the EW precision measurements and this allows a $\Lambda$ of more or less $2 \mathrm{TeV}$ when the light Higgs boson has a mass $m_{h}=115 \mathrm{GeV}$. The twin doublets model [13] is a particular version of the 2HDM in which only one doublet couples to the SM fermions. The symmetry of the model makes possible to improve the bound found in the $\mathrm{BH}$ model and to reach a cut off between 3 and $4 \mathrm{TeV}$. Finally, the inert doublet model (IDM) [12] proposes a different picture. Instead of trying to justify through naturalness the existence of a light Higgs boson and a cut off of few $\mathrm{TeV}$, it describes the possibility of having a heavy Higgs while maintaining compatibility with EW precision measurements. The cut off of the model turns out to be of few $\mathrm{TeV}$ (a value that would be natural even in the SM context if the Higgs boson were heavy). The new feature of the IDM is that the model may be compatible with the EW precision measurements even in the presence of a heavy Higgs boson. This is realized thanks to the contribution to the EW parameters that arises from the heavy new scalars. In general, in the different realizations of the $2 \mathrm{HDM}$ the $T$ parameter receives a SM-like contribution and a contribution that arises from loops involving the new scalars. These contributions are approximately given by [14]

$$
\begin{aligned}
T_{a}= & -\frac{3}{16 \pi \cos \theta_{W}^{2}}\left(\cos ^{2}(\alpha-\beta) \log \frac{m_{h}^{2}}{m_{Z}^{2}}\right. \\
& \left.+\sin ^{2}(\alpha-\beta) \log \frac{m_{H}^{2}}{m_{Z}^{2}}\right) \\
T_{b}= & \frac{1}{4 \pi s_{W}^{2} m_{W}^{2}}\left(\cos ^{2} \alpha\left(m_{H^{+}}-m_{h}\right)^{2}+\sin ^{2} \alpha\left(m_{H^{+}}-m_{H}\right)^{2}\right. \\
& +\left(m_{H^{+}}-m_{A}\right)^{2}-\cos ^{2} \alpha\left(m_{A}-m_{h}\right)^{2} \\
& \left.-\sin ^{2} \alpha\left(m_{A}-m_{H}\right)^{2}\right),
\end{aligned}
$$

where $\tan \beta=v_{2} / v_{1}$ with $v_{i}$ the VEV of $h_{i}$ and $\alpha$ the mixing angle between the two neutral scalars. If both the doublets acquire a VEV (BH, twin and the just-so models) $T_{b}$ is negligible because $m_{A}-m_{H^{+}}$cannot be too large (for natural choice of the $\lambda_{i}$ parameter of the potential). On the contrary, in the IDM $T_{b}$ may not be negligible and can balance the contribution to $T_{a}$ arising from a heavy Higgs boson; in this way, the model predicts a heavy Higgs boson and a cut off around $3 \mathrm{TeV}$. In conclusion, in all the version of $2 \mathrm{HDM}$ the cut off can be around $5 \mathrm{TeV}$ but not much higher.

Our approach is different with respect to the models that present improved naturalness. The cancellation of the Veltam condition fixes our cut off at $10 \mathrm{TeV}$ while the requirement of compatibility with the EW precision measurements forces us to include at least a new fermion doublet.

\section{ACKNOWLEDGMENTS}

This work is partially supported by MIUR and the RTN European Program MRTN-CT-2004-503369. F. B. is supported by a MEC postdoctoral grant. 
[1] S. Eidelman et al., Phys. Lett. B 592, 1 (2004).

[2] M. J. G. Veltman, Acta Phys. Pol. B 12, 437 (1981).

[3] See, for instance, M. E. Peskin and J. D. Wells, Phys. Rev. D 64, 093003 (2001).

[4] J. A. Bagger, A. F. Falk, and M. Swartz, Phys. Rev. Lett. 84, 1385 (2000).

[5] M.E. Peskin and T. Takeuchi, Phys. Rev. D 46, 381 (1992).

[6] G. Marandella, C. Schappacher, and A. Strumia, Phys. Rev. D 72, 035014 (2005).

[7] P. Gondolo, J. Edsjo, P. Ullio, L. Bergstrom, M. Schelke, and E. A. Baltz, J. Cosmol. Astropart. Phys. 07 (2004) 008.

[8] F. Bazzocchi, M. Fabbrichesi, and M. Piai, Phys. Rev. D 72, 095019 (2005).
[9] F. Bazzocchi and M. Fabbrichesi, Phys. Rev. D 70, 115008 (2004); Nucl. Phys. B715, 372 (2005).

[10] N. Arkani-Hamed, A. G. Cohen, and H. Georgi, Phys. Lett. B 513, 232 (2001); N. Arkani-Hamed, A. G. Cohen, E. Katz, A.E. Nelson, T. Gregoire, and J. G. Wacker, J. High Energy Phys. 08 (2002) 021; I. Low, W. Skiba, and D. Smith, Phys. Rev. D 66, 072001 (2002).

[11] R. Barbieri and L. J. Hall, hep-ph/0510243.

[12] R. Barbieri, L. J. Hall, and V. S. Rychkov, Phys. Rev. D 74, 015007 (2006).

[13] Z. Chacko, H. S. Goh, and R. Harnik, J. High Energy Phys. 01 (2006) 108.

[14] J. A. Casas, J. R. Espinosa, and I. Hidalgo, J. High Energy Phys. 11 (2004) 057. 